\title{
Distributed high-temperature pressure sensing using air-hole microstructural fibers
}

\author{
Tong Chen, ${ }^{1}$ Qingqing Wang, ${ }^{1}$ Rongzhang Chen, ${ }^{1}$ Botao Zhang, ${ }^{1}$ Charles Jewart, ${ }^{1}$ Kevin P. Chen,,${ }^{1, *}$ \\ Mokhtar Maklad, ${ }^{2}$ and Phillip R. Swinehart ${ }^{2}$ \\ ${ }^{1}$ Department of Electrical Engineering, University of Pittsburgh, Pittsburgh, Pennsylvania 15261, USA \\ ${ }^{2}$ Lake Shore Cryotronics, 575 McCorkle Boulevard, Westerville, Ohio 43082, USA \\ ${ }^{*}$ Corresponding author: kchen@engr.pitt.edu
}

Received October 17, 2011; revised December 9, 2011; accepted December 25, 2011; posted January 19, 2012 (Doc. ID 156643); published March 12, 2012

\begin{abstract}
We present spatially resolved Rayleigh scattering measurements in different polarization-maintaining (PM) fibers for high-temperature pressure sensing. The pressure-induced birefringence in the fiber cores is interrogated using polarization-resolved frequency-swept interferometry. The pressure responses of a PM photonic crystal fiber and a twin-air-hole PM fiber are investigated for a pressure range of 0 to $13.8 \mathrm{MPa}(0-2000 \mathrm{psi})$ at room temperature and at temperatures as high as $800{ }^{\circ} \mathrm{C}$. The proposed sensing system provides, for the first time to our knowledge, a truly distributed pressure-sensing solution for high-temperature applications. () 2012 Optical Society of America OCIS codes: $\quad 060.2370,060.4005,120.5475,120.6780$.
\end{abstract}

High-temperature pressure sensing is a challenging but indispensible task for a wide spectrum of applications in energy and aerospace industries. It plays a critical role in ensuring safe and efficient operations of fossil-fuel and nuclear power generation systems.

For sensing at high temperatures beyond $800{ }^{\circ} \mathrm{C}$, fiber sensors are probably the only option due to their excellent thermal resistivity and immunity to electromagnetic noise and corrosion. In the past few years, point sensors such as Fabry-Perot interferometry devices have been successfully developed for high-temperature applications [ $1-3$ ]. A more challenging issue is to develop multiplexing technology so the entire power system can be monitored using a single fiber. This is very important given the challenging of wiring tens or hundreds of sensors for extreme environment applications. Quasi-distributed pressure sensing can be realized with fiber Bragg gratings (FBGs) multiplexed in pressure-sensitive fibers. However, FBG-based distributed sensing capability is fundamentally limited by the multiplexing density of the FBGs and the consequent high manufacturing cost. Moreover, special grating treatments such as femtosecond laser direct writing and grating regeneration are needed to elevate the survival temperature of FBGs [ $\underline{4}-\underline{5}]$.

In this Letter, we reported for the first time truly distributed pressure sensing at room and high temperature using pressure-sensitive fibers. It is based on the optical frequency domain reflectometry measurement of in-fiber Rayleigh scattering. This technology has been applied to provide a distributed sensing solution for temperature, axial strain, and transverse stress measurements [ $\underline{6}-\underline{8}]$. In this Letter, air-hole microstructural fibers are used to extend its application for pressure measurement at high temperature. By simultaneously measuring the in-fiber Rayleigh scattering of two orthogonal polarizations, hydrostatic gas pressure applied upon the fiber under test (FUT) are spatially interrogated in subcentimeter resolution over several meters length of optical fibers. Pressure distributions of up to $13.8 \mathrm{MPa}(2000 \mathrm{psi})$ are measured in a temperature range from $24^{\circ} \mathrm{C}$ to $800{ }^{\circ} \mathrm{C}$. The technique demonstrated in this Letter completely eliminates the use of high-temperature stable FBG sensors and the challenge and cost associated with the manufacturing process.

Two polarization-maintaining (PM) pressure-sensitive fibers were investigated in this paper. Fiber A is an all-silica PM photonic crystal fiber (Blaze Photonics PM-1550-01), with pitch $\Lambda=4.4 \mu \mathrm{m}$, hole diameter $d=2.2 \mu \mathrm{m}$, and large hole diameter $d_{1}=4.5 \mu \mathrm{m}$. The cladding diameter of the fiber is $125 \mu \mathrm{m}$, and the diameter of the holey area is $\sim 40 \mu \mathrm{m}$. The microscopic image of the fiber cross section is shown in Fig. 1(a). Due to the anisotropic geometry of the holey structure around the pure silica core, the fiber birefringence in the core area is sensitive to transverse stress and external pressure. The pure silica core ensures an ultralow thermaloptic coefficient and ultrahigh operating temperature, both of which are ideal for high-temperature sensing applications [1]. Fiber B is a $220 \mu \mathrm{m}$ diameter side-hole fiber with two $90 \mu \mathrm{m}$ air holes, as shown in Fig. 1(b). The fiber core is $9.7 \mu \mathrm{m} \times 7.5 \mu \mathrm{m}$ in size and $4.5 \mu \mathrm{m}$ away from the
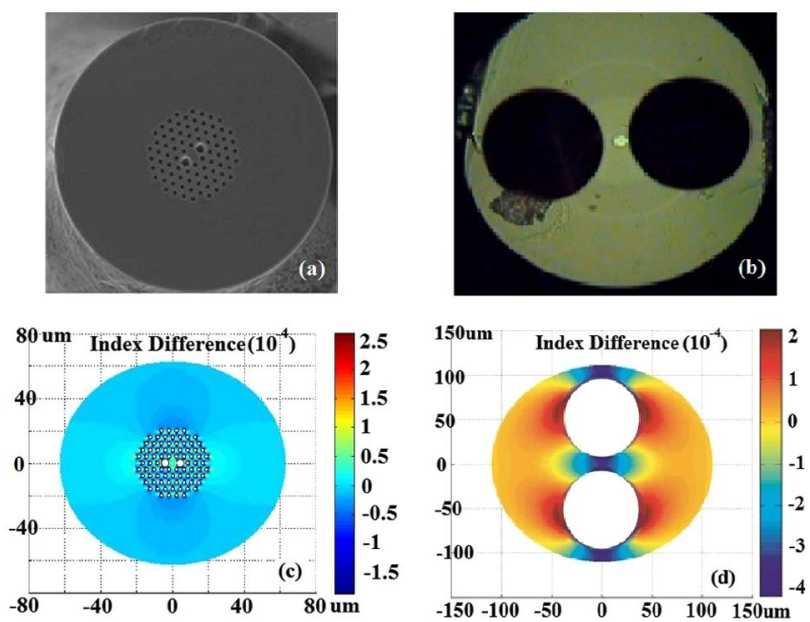

Fig. 1. (Color online) Microscope cross-section image of (a) the PM photonic crystal fiber (fiber A [9]) and (b) the twin-air-hole fiber (fiber B); finite-element analysis (FEA) simulations on 200 bar pressure-induced birefringence of (c) fiber A and (d) fiber B. 
geometric center of the fiber. When external pressure is applied, the large twin-hole structure focuses the induced birefringence into the bridge area, which significantly enhances the pressure sensitivity of fiber B compared with fiber A. Fiber B has also proved its long-term stability in temperatures as high as $850{ }^{\circ} \mathrm{C}[4-5]$. The lower two subfigures in Fig. 1 show the finite element analysis (FEA, ANSYS) simulation of pressure-induced birefringence for both fibers under $20 \mathrm{MPa}$ (2900 psi). According to the simulation, the calculated pressure sensitivity of fiber $\mathrm{B}$ is $\sim 12.0 \mu \varepsilon / \mathrm{MPa}(0.083 \mu \varepsilon / \mathrm{psi})$ or $\sim 18.7 \mathrm{pm} / \mathrm{MPa}$ $0.129 \mathrm{pm} / \mathrm{psi}$ ) at $1550 \mathrm{~nm}$, which is approximately four to five times higher than the value for fiber A. Via further optimization of the fiber holey structure, it is possible to further elevate the pressure sensitivity to $\sim 1 \mathrm{pm} / \mathrm{psi}$, so that 1 psi resolution can be achieved with a commercial tunable laser source with $1 \mathrm{pm}$ resolution at $1550 \mathrm{~nm}$ [9].

Proof-of-concept experiments were first performed at room temperature $\left(24^{\circ} \mathrm{C}\right)$ for both fibers using a commercial optical backscatter reflectometer (OBR) from Luna Technologies (OBR 4600). A tunable laser source that continuously sweeps from 1520 to $1610 \mathrm{~nm}$ is split before launch into the FUT. The excited Rayleigh scattering and possible discrete reflections in the FUT are reflected back and recombined with the split reference light onto photodiodes with a sampling rate of $2 \mathrm{MS} / \mathrm{s}$. For each Rayleigh scattering location along the FUT, the path difference between the two arms results in wavelength difference upon the mixing, and a unique beating signal is generated with location information en-
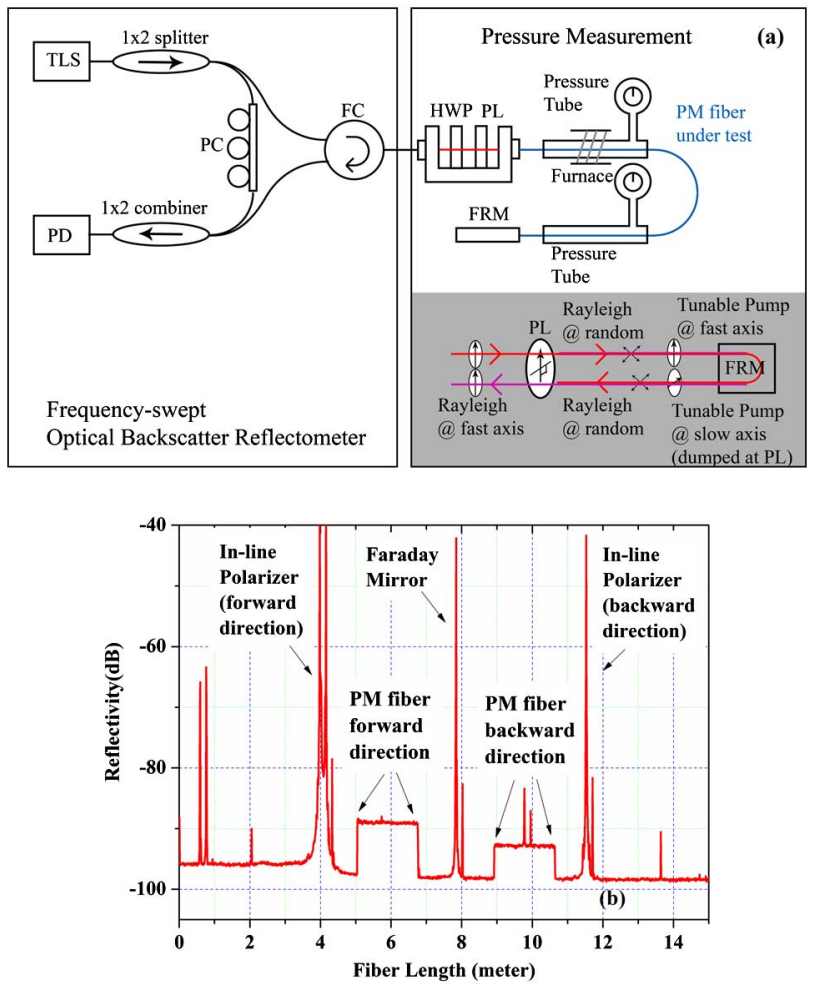

Fig. 2. (Color online) Schematic of the experimental setup and measured reflectivity symmetric to the FRM along the fiber: TLS, tunable laser source; PD, photodiode; FC, fiber circulator; HWP, half-wave plate; PL, polarizer; FRM, Faraday rotation mirror; PM fiber, polarization-maintaining fiber. coded. After the wavelength-swept measurement, this encoding can be retrieved through a fast Fourier transform upon the sweeping time/wavelength [6] . By crosscorrelating with a premeasured reference, $\bar{R}$ Rayleigh spectral shifts induced by external perturbations along the FUT can be spatially interrogated. The spatial resolution is determined by the wavelength sweeping range and the size of the cross-correlating window [ㄱ-8]. A $1 \mathrm{~cm}$ crosscorrelating window is chosen to obtain $\sim 1 \mathrm{pm}$ spectral resolution.
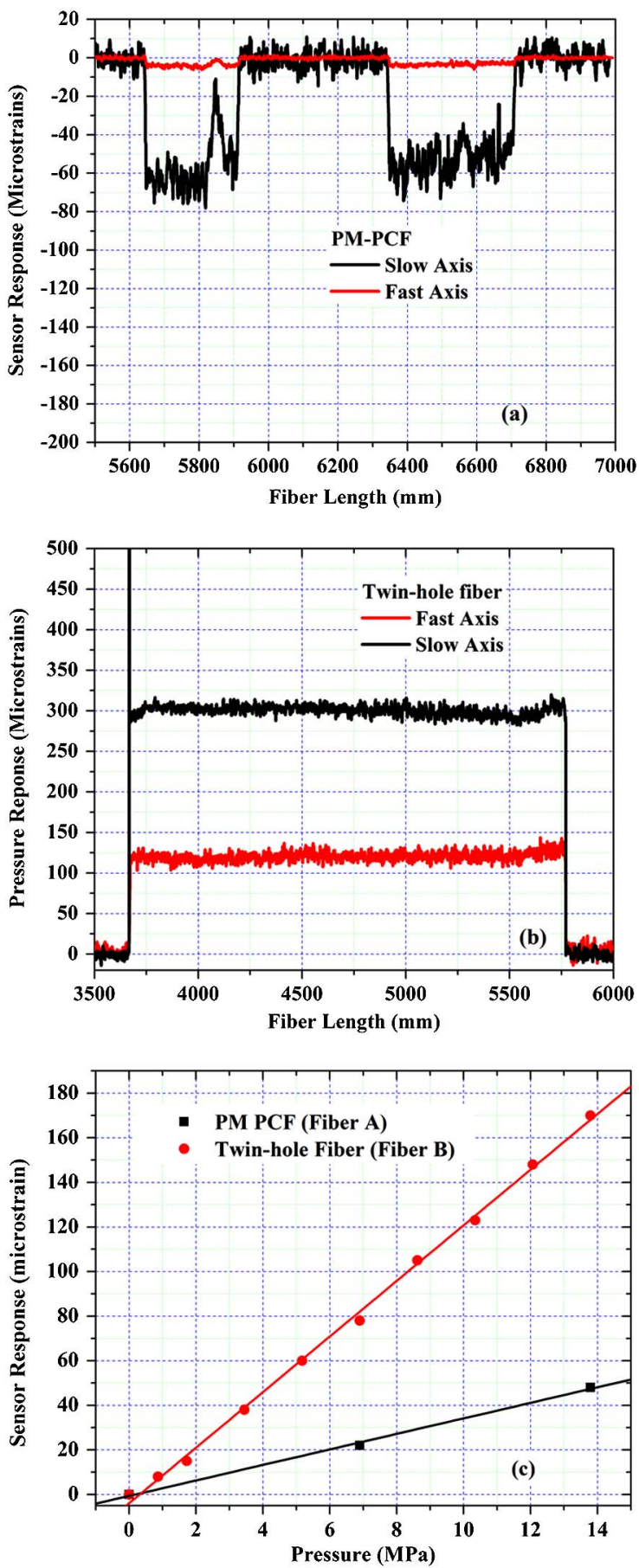

Fig. 3. (Color online) $13.8 \mathrm{MPa}(2000 \mathrm{psi})$ distributed pressure responses of (a) fiber A and (b) fiber B. (c) Induced birefringence of fiber $\mathrm{A}$ and fiber $\mathrm{B}$ versus different pressure at room temperature of $24{ }^{\circ} \mathrm{C}$. 

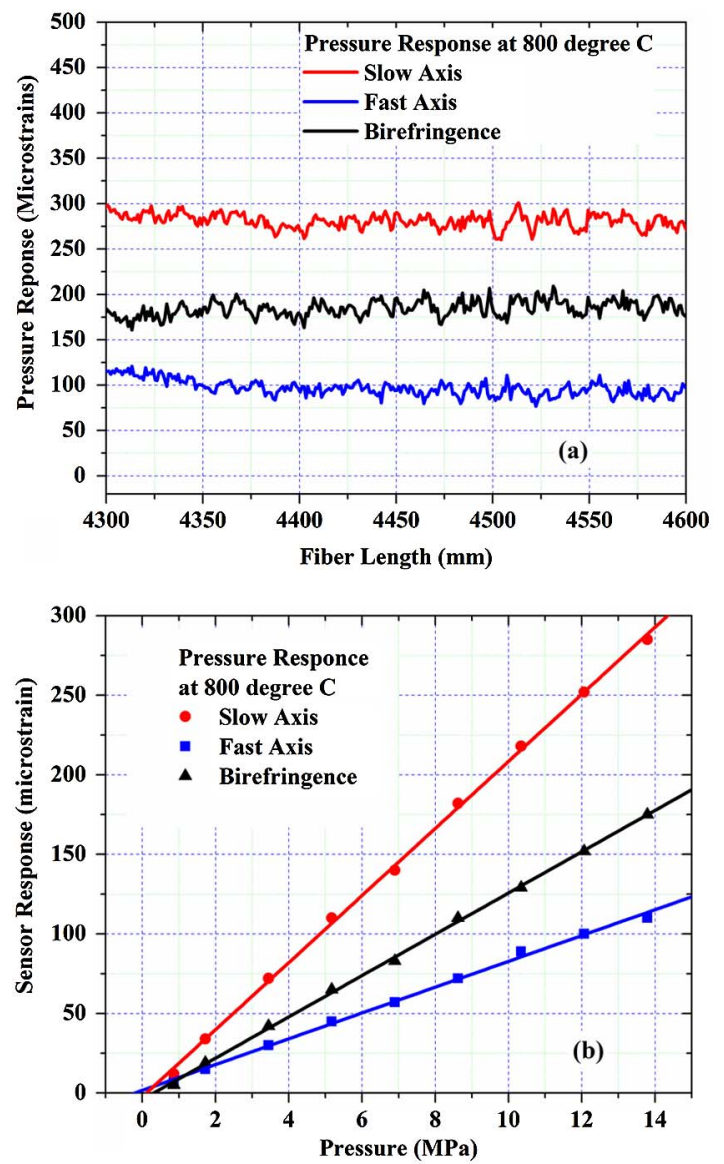

Fig. 4. (Color online) (a) $13.8 \mathrm{MPa}$ (2000 psi) distributed response of twin-hole fiber (fiber B) at $800{ }^{\circ} \mathrm{C}$ and (b) the Rayleigh sensor responses versus different pressure at $800{ }^{\circ} \mathrm{C}$.

The schematic of the experimental setup is shown in Fig. 2(a). Polarization of the tunable pump signal is aligned with the fast axis of the pressure-sensitive PM fibers using a half-wave plate and a linear polarizer. The other end of the PM fiber is fusion spliced with a Faraday rotation mirror (FRM) so that the polarization of the tunable pump signal in backward propagation is aligned with the slow axis of the PM fiber, and this slow-axis pump light is fully attenuated by the linear polarizer on the way back to the OBR to avoid any interference with the Rayleigh signal. In this configuration, Rayleigh scattering excited by both polarizations along the FUT can be measured within a single scan [10], as is shown in the OBR trace in Fig. 2(b). Birefringence responses at a certain PM fiber location can be interrogated by folding the trace along the symmetric axis at the FRM.

The room-temperature pressure responses of both fibers are measured from 0 to $13.8 \mathrm{MPa}(0-2000 \mathrm{psi})$, and the results are shown in Fig. 3. Pressure-induced birefringence is calculated from the difference between Rayleigh spectral shifts of two orthogonal polarizations. The pressure sensitivity of fiber A is $3.48 \mu \varepsilon / \mathrm{MPa}$ $(0.024 \mu \varepsilon / \mathrm{psi})$ or $5.51 \mathrm{pm} / \mathrm{MPa}(0.038 \mathrm{pm} / \mathrm{psi})$ at the central sweeping frequency of $1565 \mathrm{~nm}$. Two pressure distribution regions are measured simultaneously, showing the distributed sensing capability. Pressure sensitivity of fiber $\mathrm{B}$ is $12.5 \mu \varepsilon / \mathrm{MPa}(0.086 \mu \varepsilon / \mathrm{psi})$ or $19.6 \mathrm{pm} / \mathrm{MPa}$ $(0.135 \mathrm{pm} / \mathrm{psi})$ at $1565 \mathrm{~nm}$, which is 3.6 times larger than that of fiber A and $<5 \%$ larger than the calculated value. When the spatial resolution of the cross-correlation process is set at $1 \mathrm{~cm}$, the pressure resolution of fiber $\mathrm{B}$ is $\sim 7.4$ psi. Higher pressure resolution is available for more relaxed spatial resolution requirements.

The high-temperature pressure response of fiber $\mathrm{B}$ is investigated within a high-temperature furnace. The heating region inside the furnace is approximately $50 \mathrm{~cm}$ long. Figure 4(a) shows the spatially resolved Rayleigh spectral shifts for both fast and slow axis polarizations and the derived birefringence at $800{ }^{\circ} \mathrm{C}$. It is observed that the pressure-induced birefringence is immune to the temperature-induced fluctuations in the fast and slow axis. Figure 4(b) shows the Rayleigh spectral response of the slow axis and fast axis and the birefringence versus different pressures from 0 to $13.8 \mathrm{MPa}$ at $800{ }^{\circ} \mathrm{C}$. Good linearity of pressure dependence was observed.

In summary, we present a true distributed fiberoptic sensing technique for high-temperature pressure measurement. Polarization-resolved Rayleigh scattering signals are interrogated along several-meter-long pressure-sensitive PM fibers to obtain subcentimeter sensing resolution for pressures up to $13.8 \mathrm{MPa}$ (2000 psi) and temperatures as high as $800{ }^{\circ} \mathrm{C}$. The simplicity, low cost, and high fidelity of this technique make it a unique solution to high-temperature sensing applications.

This work was supported by the National Science Foundation (CMMI-0644681 and CMMI-0900564) and the Department of Energy (DE-FE0003859).

\section{References}

1. H. Y. Fu, C. Wu, M. L. V. Tse, L. Zhang, K. D. Cheng, H. Y. Tam, B. Guan, and C. Lu, Appl. Opt. 49, 2639 (2010).

2. C. Wu, H. Y. Fu, K. K. Qureshi, B. Guan, and H. Y. Tam, Opt. Lett. 36, 412 (2011).

3. J. Yi, E. Lally, A. Wang, and Y. Xu, IEEE Photon. Technol. Lett. 23, 9 (2011).

4. C. M. Jewart, Q. Wang, J. Canning, D. Grobnic, S. J. Mihailov, and K. P. Chen, Opt. Lett. 35, 1443 (2010).

5. T. Chen, R. Chen, C. Jewart, B. Zhang, K. Cook, J. Canning, and K. P. Chen, Opt. Lett. 36, 3542 (2011).

6. B. J. Soller, D. K. Gifford, M. S. Wolfe, and M. E. Froggatt, Opt. Express 13, 666 (2005).

7. S. T. Kreger, D. K. Gifford, M. E. Froggatt, B. J. Soller, and M. S. Wolfe, in Optical Fiber Sensors, OSA Technical Digest (CD) (Optical Society of America, 2006), paper ThE42.

8. R. R. J. Maier, W. N. MacPherson, J. S. Barton, S. McCulloch, and B. J. S. Jones, Meas. Sci. Technol. 21, 094019 (2010).

9. http://www.thorlabs.com/Thorcat/8700/8767-M01.pdf.

10. C. Jewart, S. M. Quintero, A. M. B. Braga, and K. P. Chen, Opt. Express 18, 25657 (2010). 